\title{
PET in Infancy Predicts Long-Term Outcome during Adolescence in Cryptogenic West Syndrome
}

\author{
J. Natsume, N. Maeda, K. Itomi, H. Kidokoro, N. Ishihara, H. Takada, A. Okumura, T. Kubota, K. Miura, K. Aso,
}

T. Morikawa, K. Kato, T. Negoro, and K. Watanabe

\begin{abstract}
BACKGROUND AND PURPOSE: Developmental and seizure outcomes in patients with cryptogenic West syndrome are variable. Our aim was to clarify the relationship between FDG-PET findings in infancy and long-term seizure and developmental outcome in cryptogenic West syndrome.

MATERIALS AND METHODS: From 1991 to 1999, we prospectively performed FDG-PET from the onset of cryptogenic West syndrome in 27 patients. PET was performed at onset and at 10 months of age. In 2012, we evaluated the educational status, psychomotor development, and seizure outcome in 23 of the 27 patients (13-22 years of age). The correlation between PET findings and outcome was evaluated.

RESULTS: At onset, PET showed hypometabolism in 13 patients (57\%). The second PET after the initial treatment revealed cortical hypometabolism in 7 patients (30\%). While hypometabolism at onset disappeared on the second PET in 9 patients, it was newly revealed in 3 patients on the second PET. In 2012, seven patients had persistent or recurrent seizures. Eight patients had intellectual impairment. The first PET did not correlate with seizure or developmental outcome. Five of 7 patients (71\%) with hypometabolism seen on the second PET had persistent or recurrent seizures, while 14 of 16 (88\%) patients with normal findings on the second PET were free of seizures. Five of 7 patients (71\%) showing hypometabolism on the second PET had intellectual impairment. Thirteen of 16 (81\%) patients with normal findings on the second PET showed normal intelligence. A significant correlation was found between the second PET and long-term seizure $(P=.01)$ or developmental outcome $(P=.03)$.
\end{abstract}

CONCLUSIONS: Cortical hypometabolism is not permanent; it changes with clinical symptoms. Hypometabolism after initial treatment predicts long-term seizures and poor developmental outcome.

ABBREVIATIONS: $\mathrm{ACTH}=$ adrenocorticotropic hormone; $\mathrm{DQ}=$ developmental quotient; $\mathrm{EEG}=$ electroencephalography; $\mathrm{IQ}=$ intelligence quotient

W est syndrome is an age-dependent epileptic encephalopathy characterized by epileptic spasms and the specific interictal electroencephalography (EEG) abnormality of hypsarrhythmia. Patients with West syndrome often show deterioration of psychomotor development after the onset. Although the long-

Received September 16, 2013; accepted after revision November 28.

From the Departments of Pediatrics (J.N., N.M., H.K., N.I., H.T., K.M., T.N.) and Radiological and Medical Laboratory Sciences (K.K.), Nagoya University Graduate School of

Medicine, Nagoya, Japan; Department of Neurology (K.I.), Aichi Children's Health and Medical Center, Obu, Japan; Department of Pediatrics (A.O.), Juntendo University Faculty of Medicine, Tokyo, Japan; Department of Pediatrics (T.K.), Anjo Kosei Hospital, Anjo, Japan; Department of Pediatrics (K.A.), Aichi Prefecture Medical Welfare Center of Aoitori, Nagoya, Japan; Morikawa Clinic (T.M.), Nagoya, Japan; and Faculty of Health and Medical Sciences (K.W.), Aichi Shukutoku University, Nagakute, Japan.

Please address correspondence to Jun Natsume, MD, PhD, Department of Pediatrics, Nagoya University Graduate School of Medicine, 65 Tsurumai-cho, Showa-ku, Nagoya, Aichi 466-8550, Japan; e-mail: junnatsu@med.nagoya-u.ac.jp

- Indicates open access to non-subscribers at www.ajnr.org

EIndicates article with supplemental on-line table.

http://dx.doi.org/10.3174/ajnr.A3899 term outcome is better in patients with cryptogenic etiology, the developmental and seizure outcomes in cryptogenic patients are variable. $^{1-3}$

Since the description by Chugani et al, ${ }^{4,5}$ the use of PET to detect hypometabolism in cryptogenic West syndrome has come into the spotlight. We and others have reported PET hypometabolism or SPECT hypoperfusion during the early stage of cryptogenic West syndrome. ${ }^{6-11}$ We also have reported evolutional changes in cortical hypometabolism on PET and have suggested the usefulness of PET for predicting the prognosis. ${ }^{6-9}$ The results of our studies and others revealed that persistent hypometabolism or hypoperfusion after initial pharmacologic treatment suggests poor developmental outcome. ${ }^{6-9,11,12}$ However, the follow-up period of these studies was limited to a minimum of 2 or 3 years. Developmental statuses and seizures in school age or adulthood should be investigated to reveal long-term outcome.

In the present study, we investigated the educational and occupational statuses, psychomotor development, and seizure 
outcome in patients with cryptogenic West syndrome who underwent prospective serial PET scans from the onset in our previous studies and were now 13 years of age or older.

\section{MATERIALS AND METHODS}

Between April 1991 and December 1999, we prospectively registered all 27 children (16 boys) with new-onset cryptogenic West syndrome who were admitted to Nagoya University Hospital and performed PET studies. The criteria for diagnosing cryptogenic West syndrome were as follows: 1) normal birth and absence of any etiologic factors related to West syndrome, 2) normal development before the onset and absence of neurologic abnormalities at the onset, 3) the occurrence of clusters of spasms without any other types of seizures before the onset of spasms, and 4) normal laboratory and MR imaging findings at onset. We excluded patients who had epilepsy surgery in infancy. The term "cryptogenic" was replaced by "unknown" by the International League Against Epilepsy Commission on Classification and Terminology in $2010 .{ }^{13}$ However, the definition of cryptogenic here includes seizure types and development and unknown etiology. Therefore, we decided to use the term "cryptogenic" in this study.

FDG-PET was performed twice. The first scans were obtained at the onset of spasms before adrenocorticotropic hormone (ACTH) therapy, and the second scans were obtained at 10 months of age. The first scans were obtained at 4-8 months of age except in 2 patients who were diagnosed and scanned at 13 and 28 months of age. The second scans were obtained $>1$ month after the administration of ACTH therapy to avoid the effect of brain shrinkage by ACTH. In the 2 patients who were diagnosed after 12 months of age, the second scans were obtained 3 months after the initial treatment, at 16 months of age in one patient and at 31 months of age in the other. The scans were obtained as part of the clinical routine to search for underlying pathology. Before the PET scan, we explained to the parents that the results would be used for research, and informed consent was obtained from the parents.

All patients were sedated by chloral hydrate suppository $(30-50 \mathrm{mg} / \mathrm{kg})$ during the PET examination. Fifty minutes after intravenous administration of FDG (36-111 MBq), 14 axial images (11-mm thick) were obtained with a Headtome IV scanner (Shimadzu, Kyoto, Japan) with spatial resolution at 6.51-mm intervals parallel to the orbitomeatal line, starting from the orbitomeatal line $+0 \mathrm{~mm}$. PET images were analyzed with visual inspection by 3 pediatric neurologists (J.N., N.M., and K.I). All 3 pediatric neurologists were regularly involved in neuroimaging studies and had experience in the interpretation of pediatric PET images. We referred to the PET study in infants by Chugani et $\mathrm{al}^{14}$ to evaluate the PET findings correctly.

"Regional hypometabolism" was defined as a regional decrease in FDG accumulation in $\geq 2$ gyri on $\geq 2$ sections. Diffuse hypometabolism was considered present when there was a decrease in the entire cerebral cortex compared with accumulation in the basal ganglia. We did not use quantitative methods because measuring absolute glucose metabolic rates with arterial blood sampling during the scan is difficult in infants who are kept still. We also did not use a coregistration method of PET and MR imaging because myelination is not complete in the early infantile period and we could not apply coregistration programs that use T1-weighted images with completed myelination.

Patients were initially treated by the following protocol: Sodium valproate, clonazepam, or pyridoxine was administered for 1 week. If these agents failed to control the spasms, thyrotropinreleasing hormone was administered intramuscularly for 2 weeks. If thyrotropin-releasing hormone could not control the spasms, ACTH therapy was started. Since 1998, we have excluded thyrotropin-releasing hormone therapy from our protocol because it was found to be less effective than ACTH therapy. In the present study, 5 patients (patients 19-23) did not receive thyrotropinreleasing hormone therapy. In the present study, synthesized ACTH was injected intramuscularly at $0.015 \mathrm{mg} / \mathrm{kg} /$ day for $2-4$ weeks followed by alternate-day administration for 1 week. The effectiveness of each drug was evaluated on the basis of the frequency and intensity of spasms and the interictal EEG findings.

MRIs were repeated at 10 months of age in all patients and at 2-16 years of age in 17 patients to reveal structural lesions that had not been detected at the onset. We rereviewed the MRIs for subtle structural abnormality if the MRIs were available in 2012.

Evaluation of developmental and seizure outcome was performed between August and December 2012. The age at the evaluation ranged from 13 to 22 years (median, 17 years). The information was obtained from the medical charts at Nagoya University or affiliated hospitals. In cases in which the follow-up was terminated, we interviewed the parents by questionnaire after informed consent was obtained. We determined educational status and intelligence quotient (IQ) or developmental quotient (DQ). The IQ was measured by the Wisconsin Intelligence Scale for Children or the Tanaka-Binet Intelligence Scale, and DQ was measured by the Tsumori-Image Developmental Questionnaire. Developmental status was considered as follows: 1) normal, when patients attended regular classes at a regular school or had an IQ of 70 or greater; 2) mild intellectual disability, when patients attended a special needs class at a regular primary school or had an IQ between 50 to 69; and 3) severe intellectual disability, when patients attended a school for handicapped children during primary school or had an IQ of $<50$. We also determined from the medical chart whether the patients were diagnosed with autism. Seizure outcome was categorized as follows: 1) free of seizures, when patients had no seizures since 2 years of age, except for simple febrile seizures, and were off antiepileptic medications in 2012; and 2) persistent or recurrent seizures, when patients continued to have epileptic seizures after 2 years of age and were on antiepileptic medications.

The present study was performed continuously from our previous studies. ${ }^{6-9}$ We added 9 patients to the previous report by Itomi et $\mathrm{al}^{9}$ and excluded 3 patients who were out of contact in 2012.

The Fisher exact test was used for statistical analysis of the correlation between hypometabolism at onset or second scans and seizure or developmental outcome. Significance was established at $P<.05$. We calculated positive and negative predictive values of the PET studies.

This study was approved by the research ethics committee of Nagoya University Graduate School of Medicine.

AJNR Am J Neuroradiol 35:1580-85 Aug 2014 www.ajnr.org 1581 


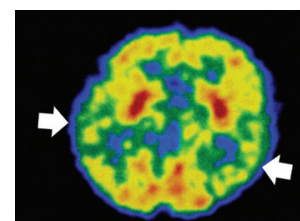

6 mo

1) Patient 12

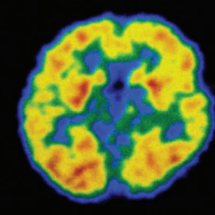

$10 \mathrm{mo}$

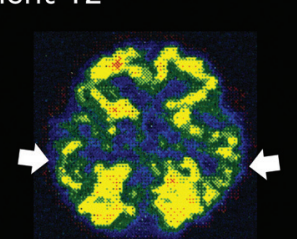

$10 \mathrm{mo}$

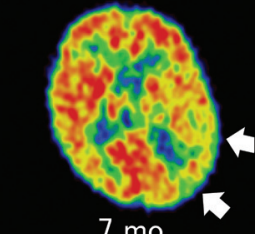

$7 \mathrm{mo}$
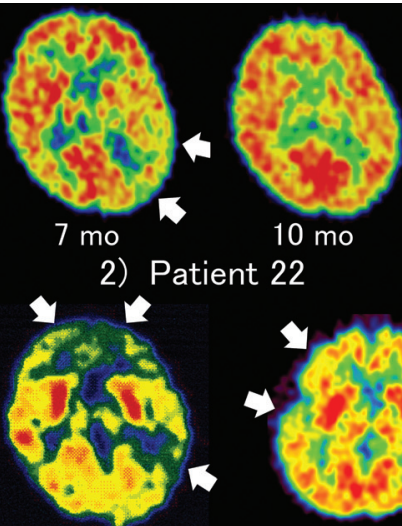

$10 \mathrm{mo}$
22

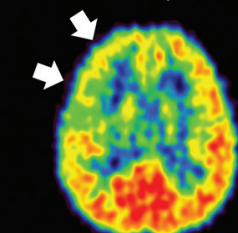

$10 \mathrm{mo}$
4) Patient 11

3) Patient 20

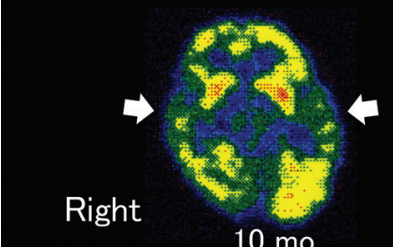

$10 \mathrm{mo}$

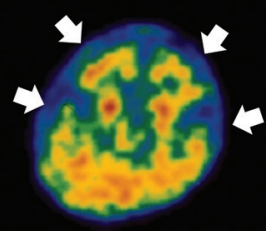

10 mo

8) Patient 17 tient 13

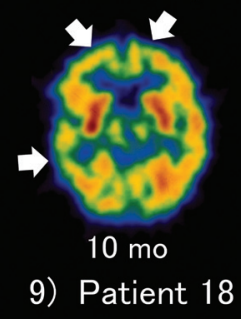

FIG 1. Hypometabolism as seen on the first and second PET scans. 1) Patient 12: PET at 6 months of age reveals bilateral temporal hypometabolism. Hypometabolism is resolved at 10 months of age. The patient is free of seizures without medication and in normal high school at 17 years of age. 2) Patient 22: PET at 7 months of age shows left temporal hypometabolism that is resolved at 10 months of age. The patient is free of seizures without medication and in a normal high school at 13 years of age, though he is diagnosed with Asperger syndrome. 3) Patient 20: PET at 10 months of age shows right frontal hypometabolism. The patient is free of seizures without medication and in a normal high school at 14 years of age. 4) Patient 11: PET at 10 months of age reveals bitemporal hypometabolism. The patient experienced a relapse of spasms at 16 months of age and continues to have partial seizures since 4 years of age and mild intellectual disability. 5) Patient 13: PET at 10 months of age reveals multifocal hypometabolism in the bilateral frontal lobes and right temporal lobe. The patient has severe intellectual impairment. 6) Patient 21: PET at 10 months of age shows bifrontotemporal hypometabolism. The patient has persistent seizures and severe intellectual impairment. 7) Patient 14: PET at 10 months of age reveals bitemporal hypometabolism. The patient is free of seizures except for febrile seizures at 3 years of age and attends normal high school. 8) Patient 17: PET at 10 months of age shows bifrontotemporal hypometabolism. The patient has experienced partial seizures since 10 years of age and has severe intellectual impairment. 9) Patient 18: PET at 10 months of age shows bifrontal and right temporal hypometabolism. The patient has spasms, tonic seizures, atypical absence seizures, and severe intellectual impairment.

\section{RESULTS}

Twenty-seven patients with cryptogenic West syndrome underwent serial PET during the study period. In 2012, information could not be obtained from 3 patients who were out of contact. We also excluded 1 patient because MR imaging at 2 years of age revealed focal cortical dysplasia. Therefore, we analyzed 23 patients ( 11 boys) in whom enough clinical information was available. We rereviewed MRIs of patients for subtle structural abnormalities if the MRIs were available in 2012. In 10 patients, the MRIs were not available in 2012; therefore, prior readings were used. In the other 13 patients, we re-evaluated the MR imaging findings and interpreted them as normal except for diffuse mild atrophy in 1 patient. Our MR imaging protocol in 1991-1999 did not include modern 3D high-resolution images, and there is a possibility that subtle focal cortical dysplasia may have been missed. The results are summarized in the On-line Table. The ages at onset ranged from 3 to 26 months (median, 5 months). ACTH therapy was administered as initial treatment in 21 patients. In the other 2 patients, spasms and hypsarrhythmia disappeared with

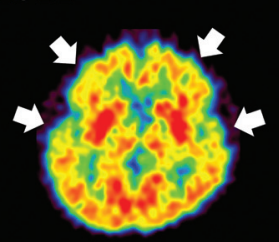

$10 \mathrm{mo}$ administration of thyrotropin-releasing hormone or valproate. The time interval from the onset of spasms to initial treatment ranged from 1 to 7 months.

\section{PET Hypometabolism}

The initial PET scans demonstrated cortical hypometabolism in 13 of 23 patients. Hypometabolism was bilateral, symmetric, and regional in 7 patients; unilateral regional in 4; and diffuse in 2 . The regions of hypometabolism were occipitotemporal or occipital parietal temporal in 3 patients, temporal or temporoparietal in 4 patients (Fig 1), parietal in 2 patients, frontotemporal in 2 patients, and diffuse in 2 patients.

The second PET showed regional hypometabolism in 7 patients. Bilateral temporal or bilateral temporofrontal hypometabolism was seen in 4 patients; unilateral frontal hypometabolism, in 1 patient; and bilateral multifocal hypometabolism, in 2 patients (Fig 1). In 9 of 13 patients with hypometabolism on the first PET, hypometabolism was normalized on the second PET. On the other hand, 3 patients with normal findings on the first scan had hypometabolism on the second PET. Three patients with hypometabolism on the second PET underwent a third PET at 1 year of age, and they still showed cortical hypometabolism. Interictal EEG at the time of the second PET showed epileptiform discharges in 9 patients. All 7 patients with hypometabolism on the second PET had EEG abnormality at the second PET. Two patients with normal second PET findings had EEG abnormalities at the second PET. In 4 of 6 patients with bilateral hypometabolism on the second PET, EEG at 10 months of age showed bilateral abnormality, including hypsarrhythmia or diffuse spike-waves. The other 2 patients (patients 11 and 14) with bitemporal hypometabolism had unilateral temporal spikes at 10 months of age. One patient with right frontal hypometabolism at 10 months of age had bilateral centro-parieto-temporal spikes. The localization of PET hypometabolism and EEG abnormality correlated anatomically, though the distribution of hypometabolism was more widespread in 2 patients and more focal in 1 patient than that of EEG abnormality. Ictal recordings of partial seizures during the follow-up period were not made because the seizures were not frequent. In 4 of 7 patients with hypometabolism on the second PET, MRIs were repeated at 24-36 months of age, and corresponding lesions were not revealed.

\section{Seizure and Developmental Outcome}

No patient had any special comorbidity except for epileptic seizures or delayed psychomotor development. After initial treat- 
Table 1: Correlations between PET hypometabolism and seizure outcome $^{\mathrm{a}}$

\begin{tabular}{lccc}
\hline & Hypometabolism & $\begin{array}{c}\text { Patients with } \\
\text { Persistent or } \\
\text { Recurrent Seizures }\end{array}$ & $\begin{array}{c}\text { Patients Free } \\
\text { of Seizures }\end{array}$ \\
\hline 1st PET & Normal & 4 & 9 \\
\multirow{3}{*}{ 2nd PET } & Normal & 3 & 7 \\
& & 5 & 2 \\
& & 2 & 14 \\
\hline
\end{tabular}

${ }^{\text {a }}$ Positive predictive value of second PET was 71\%; negative predictive value of second PET, $88 \% ; P=.01$.

Table 2: Correlations between PET hypometabolism and developmental outcome ${ }^{\mathrm{a}}$

\begin{tabular}{|c|c|c|c|}
\hline & Hypometabolism & $\begin{array}{l}\text { Patients with } \\
\text { Special } \\
\text { Education }\end{array}$ & $\begin{array}{l}\text { Patients in } \\
\text { Regular High School }\end{array}$ \\
\hline \multirow[t]{2}{*}{ 1st PET } & Normal & 4 & 9 \\
\hline & & 4 & 6 \\
\hline \multirow[t]{2}{*}{ 2nd PET } & Normal & 5 & 2 \\
\hline & & 3 & 13 \\
\hline
\end{tabular}

${ }^{a}$ Positive predictive value of second PET was 71\%; negative predictive value of second PET, $81 \% ; P=.03$.

ment, spasms disappeared in 19 patients, but 4 of the 19 patients had relapse of seizures. One patient with relapse of spasms at 9 months of age received a second round of ACTH therapy and has become free of seizures. At the final follow-up period in 2012, sixteen patients had no seizures since 2 years of age, without antiepileptic medication. The other 7 patients still had spasms, tonic seizures, atypical absences, or partial seizures and were receiving antiepileptic medication. Four of these 7 patients continued to have seizures from infancy. The other 3 patients did not have seizures from infancy, but partial seizures had occurred since 4 or 13 years of age. Regarding education, 16 patients completed their education at a normal primary school. Three patients attended special needs classes in regular primary schools, and 4 patients attended schools for handicapped children. During high school, 15 patients entered regular high schools, and 8 patients entered high schools for handicapped children. In 6 patients who completed their education at regular high schools, 4 attended college and 2 began working in an occupation after high school graduation. Two patients with intellectual disability were diagnosed with autism, and 1 patient with normal intelligence was diagnosed with Asperger syndrome.

In 16 patients, MR images were obtained between 2 and 16 years of age to see if any lesions had been overlooked, but they did not reveal any abnormality except mild atrophy in one patient. MRIs were not re-performed at 2 years of age or later in 7 patients, including 2 patients with severe intellectual disability and persistent seizures in whom sedation was difficult during scans.

\section{Correlation of PET Hypometabolism and Long-Term Outcome}

Tables 1 and 2 show correlations between hypometabolism on the first or second PET scans and long-term outcome. In 13 patients with hypometabolism on the initial PET, 4 (31\%) had persistent or recurrent seizures during the follow-up period. In 10 patients with normal initial PET findings, 3 (30\%) had persistent or relapsing seizures. No correlation was observed between the initial PET results and seizure outcome. Regarding the second PET, 5 of
7 patients with hypometabolism had persistent or recurrent seizures (positive predictive value, $71 \%$ ). In 16 patients without hypometabolism on the second PET, 14 were free of seizures without medication (negative predictive value, $88 \%$ ). A significant correlation was seen between the second PET findings and seizure outcome $(P=.01)$.

Concerning development and educational statuses, special education in high school was needed for 4 of 13 patients with hypometabolism on the initial PET and 4 of 10 patients with normal findings on the initial PET. No significant correlation was observed between initial PET results and educational status. Regarding the second PET, 5 of 7 patients with hypometabolism had intellectual impairment and required special needs education. All 4 patients who had bilateral frontal hypometabolism with or without temporal hypometabolism on the second PET had the most severe intellectual disability, with an IQ of $<20$. On the other hand, only 3 of 16 patients with normal second PET findings required special needs education. A significant correlation was seen between the second PET results and educational status $(P=.01)$. The positive predictive value of second-PET hypometabolism for poor developmental outcome was $71 \%$, and the negative predictive value was $81 \%$. In 9 patients with EEG abnormality at the time of the second PET, only 2 of 7 patients with a second PET abnormality entered regular high schools, while both of the patients with normal second PET findings entered regular high schools. In patient 22, who had Asperger syndrome, PET showed left temporal hypometabolism at onset (Fig 1). However, the hypometabolism was resolved on the second PET. At 6 years of age, EEG still showed left frontal spikes, but 3T MR imaging and PET did not show any abnormality.

\section{DISCUSSION}

We performed serial PET studies from the onset of cryptogenic West syndrome in infancy and studied the correlation between PET findings and long-term outcome in adolescence. The second PET finding after initial treatment was correlated with poor seizure and developmental outcome. On the other hand, long-term outcome was not correlated with the initial PET findings before effective treatment. This is the first report revealing the usefulness of PET performed in infancy to predict long-term outcome during adolescence in patients with cryptogenic West syndrome.

We previously reported serial PET studies in cryptogenic and symptomatic West syndrome. ${ }^{6-9}$ In our studies, PET at the onset of spasms showed cortical hypometabolism in two-thirds of patients. However, the hypometabolism was not persistent but resolved in many patients after initial treatment. Persistent cortical hypometabolism after initial treatment was correlated with poor developmental outcome in infancy and early childhood. Other investigators have confirmed these results by using SPECT or PET. ${ }^{10,11}$ In all of the previous studies, the follow-up period was limited to a minimum of 2 or 3 years, and educational outcome was not reported. Although gross motor development or simple language development can be evaluated by 2 or 3 years of age, evaluation at this age is not sufficient for higher cognitive functioning or socioeducational status. Dilber et al ${ }^{12}$ revealed a correlation between PET findings and autism in patients with West syndrome. However, the age of the study subjects varied from 3 to 16 years; therefore, the investigators could not determine whether 
PET in the infantile period is useful for predicting autism or poor psychomotor development. In the evaluation of seizures, though the cessation of spasms can be confirmed by 2 or 3 years of age, focal epilepsy often develops after cessation of spasms; a longer follow-up period is therefore needed to conclusively learn the outcome. The present study has confirmed the usefulness of PET after initial treatment to predict long-term outcome in patients with cryptogenic West syndrome.

A correlation was observed between second PET studies in the infantile period after initial treatment and long-term developmental outcome. Latent cortical dysplasia may cause persistent regional hypometabolism and may result in poor developmental outcome. The second PET showed bitemporal hypometabolism in 4 patients. Three of these patients had autism or severe intellectual impairment. This finding had been earlier reported by Chugani et $\mathrm{al}^{15}$ as a distinct phenotype characterized by developmental delay and autism. Another 2 patients had multifocal or bilateral hypometabolism, which has also been reported as a major type of PET abnormality in patients with poor outcome. ${ }^{16}$ The seventh patient had unifocal frontal hypometabolism, but her seizure and developmental outcome was favorable. Although rare, there may be some patients with focal cortical dysplasia and West syndrome in whom seizures disappear and cognitive function is preserved. The sensitivity of PET in the second scans in our series was much lower than that in the study by Chugani and Conti. ${ }^{16}$ Their study focused on patients with intractable spasms, but our study patients were enrolled prospectively from the onset and may have had characteristics similar to those of patients in the general population with cryptogenic West syndrome. On the other hand, the initial PET revealed cortical hypometabolism in more than half of the patients in our study. Most of these findings have resolved, especially in the patients with favorable seizure and developmental outcome. Reversible functional abnormalities caused by abnormal epileptic activities may cause transient cortical hypometabolism in some of these patients. From our observations, PET at the onset is not necessary for all patients, though it is useful for early surgery in patients with poor seizure control. ${ }^{4}$ Yet, as in the patient with Asperger syndrome, transient hypometabolism might be related to high-functioning developmental disorders, but the correlation with them remains to be clarified.

Indeed, the correlation between PET findings and seizure outcome was not conclusive in previous studies. While our previous report on patients with cryptogenic and symptomatic West syndrome in 1994 showed a significant correlation between the results of the second scan and seizure outcome, ${ }^{7}$ another of our reports in 2002, which focused on cryptogenic patients, did not show any correlation between PET and seizure outcome. ${ }^{9}$ The ages of the study patients in the follow-up period of our 1994 report ranged from 19 to 47 months, and the ages of the study patients in the follow-up period in our 2002 report ranged from 3 to 8 years. The difference in the follow-up periods may have been responsible for the dissimilar results. Another possible reason for the varying results among the different studies is whether symptomatic patients were included. Metsähonkala et $\mathrm{al}^{10}$ studied PET hypometabolism in cryptogenic or symptomatic patients and concluded that PET did not have prognostic value for seizure outcome. In the present study with a longer follow-up in crypto- genic patients, we found a significant correlation between PET findings in infancy and seizure outcome. However, seizure outcomes were not predicted from the second PET findings in some patients. One patient with normal findings on the first and second PET scans developed partial seizures at 13 years of age after a seizure-free period of $>10$ years. The condition of epileptic networks could change variably with maturation, and the onset of partial seizures at older ages cannot be ruled out from the PET findings in the infantile period.

Factors associated with good prognosis in West syndrome have been determined in many studies. ${ }^{1-3,17,18}$ Reported favorable factors are cryptogenic etiology, age at onset older than 4 months, no or mild mental deterioration at presentation, short treatment lag, good response to ACTH therapy, no reappearance of paroxysmal discharges on EEG, and no other types of seizures. Treatment lag has been especially emphasized because prognosis is possibly improved by prompt diagnosis and early treatment. In view of our previous study about hypometabolism and delayed myelination on MR imaging, persistent cortical hypometabolism may result in the delay of cerebral maturation and impaired cognitive function. ${ }^{8}$ Early treatment of functional abnormalities may improve the long-term developmental outcome. In the present study, 4 patients had a treatment lag of 3 months or longer, and the developmental outcome was poor in all 4 of these patients. A patient with a treatment lag of 7 months had diffuse hypometabolism at the first PET scan. Long-lasting electrographic abnormalities of hypsarrhythmia may cause widespread cerebral glucose hypometabolism and developmental delay, even with normalized metabolism, as seen at the second scan in this patient. PET at the time of initial treatment might add information about the adverse effects of a long treatment lag.

Although the response of seizures and EEG findings to initial treatments are also good markers for predicting outcome, patients 22 and 23, who had persistent EEG abnormalities after treatment, showed good seizure responses and outcomes. PET findings at 10 months were normal in these patients, and this outcome may give additional useful information for predicting long-term prognosis. Response to ACTH therapy is also a factor for favorable outcome. However, 3 patients in our study initially responded to ACTH but had a relapse of seizures and intellectual disability. One of the 3 patients with a relapse of seizures had bitemporal hypometabolism at 10 and 20 months of age, and the PET finding may suggest poor long-term outcome.

Other factors may affect educational and developmental outcomes. Concerning the duration of the follow-up period, educational and developmental outcomes did not have significant correlation with the duration of the follow-up period, though 8 patients with developmental delay tended to have a shorter follow-up period (mean, 16.6 \pm 2.1 years) than patients with normal development (mean, $17.5 \pm 3.0$ years). Treatment protocol had not changed largely except for the exclusion of thyrotropin-releasing hormone therapy from 1998, and the change of the treatment protocol did not seem to affect the long-term outcome.

\section{CONCLUSIONS}

The evaluation of hypometabolism as seen on PET during the infantile period is useful for predicting long-term seizure and de- 
velopmental outcome in patients with cryptogenic West syndrome. We suggest that PET should be performed at 10-12 months of age for early detection of underlying abnormality and prediction of long-term outcome. The hypometabolism seen at the onset often resolves; persistent hypometabolism after initial treatment is related to poor long-term outcome.

\section{ACKNOWLEDGMENTS}

The authors thank the staff of the PET units of Nagoya University Hospital.

\section{REFERENCES}

1. Riikonen R. Long-term outcome of patients with West syndrome. Brain Dev 2001;23:683-87

2. Kivity S, Lerman P, Ariel R, et al. Long-term cognitive outcomes of a cohort of children with cryptogenic infantile spasms treated with high-dose adrenocorticotropic hormone. Epilepsia 2004;45:255-62

3. Hamano S, Yoshinari S, Higurashi N, et al. Developmental outcomes of cryptogenic West syndrome. J Pediatr 2007;150:295-99

4. Chugani HT, Shields WD, Shewmon DA, et al. Infantile spasms: I. PET identifies focal cortical dysgenesis in cryptogenic cases for surgical treatment. Ann Neurol 1990;27:406-13

5. Chugani HT, Shewmon DA, Sankar R, et al. Infantile spasms: II. Lenticular nuclei and brain stem activation on positron emission tomography. Ann Neurol 1992;31:212-19

6. Maeda N, Watanabe K, Negoro T, et al. Transient focal cortical hypometabolism in idiopathic West syndrome. Pediatr Neurol 1993;9:430-34

7. Maeda N, Watanabe K, Negoro T, et al. Evolutional changes of cortical hypometabolism in West's syndrome. Lancet 1994;343:1620-23
8. Natsume J, Watanabe K, Maeda N, et al. Cortical hypometabolism and delayed myelination in West syndrome. Epilepsia 1996;37:1180-84

9. Itomi K, Okumura A, Negoro T, et al. Prognostic value of positron emission tomography in cryptogenic West syndrome. Dev Med Child Neurol 2002;44:107-11

10. Metsähonkala L, Gaily E, Rantala H, et al. Focal and global cortical hypometabolism in patients with newly diagnosed infantile spasms. Neurology 2002;58:1646-51

11. Hamano S, Yoshinari S, Higurashi N, et al. Regional cerebral blood flow and developmental outcome in cryptogenic West syndrome. Epilepsia 2007;48:114-19

12. Dilber C, Calişkan M, Sönmezoğlu K, et al. Positron emission tomography findings in children with infantile spasms and autism. J Clin Neurosci 2013;20:373-76

13. Berg AT, Berkovic SF, Brodie MJ, et al. Revised terminology and concepts for organization of seizures and epilepsies: report of the ILAE Commission on Classification and Terminology, 2005-2009. Epilepsia 2010;51:676-85

14. Chugani HT, Phelps ME, Mazziotta JC. Positron emission tomography study of human brain functional development. Ann Neurol 1987;22:487-97

15. Chugani HT, Da Silva E, Chugani DC. Infantile spasms: III. Prognostic implications of bitemporal hypometabolism on positron emission tomography. Ann Neurol 1996;39:643-49

16. Chugani HT, Conti JR. Etiologic classification of infantile spasms in 140 cases: role of positron emission tomography. J Child Neurol 1996;11:44-48

17. Riikonen RS. Favourable prognostic factors with infantile spasms. Eur J Paediatr Neurol 2010;14:13-18

18. O'Callaghan FJ, Lux AL, Darke K, et al. The effect of lead time to treatment and of age of onset on developmental outcome at 4 years in infantile spasms: evidence from the United Kingdom Infantile Spasms Study. Epilepsia 2011;52:1359-64 